\title{
Mass Transfer Effects on Unsteady Hydromagnetic Convective Flow past a Vertical Porous Plate in a Porous Medium with Heat Source
}

\author{
S. S. Das ${ }^{1 \dagger}$, S. R. Biswal ${ }^{2}$, U.K. Tripathy ${ }^{3}$ and P. Das ${ }^{1}$ \\ ${ }^{1}$ Department of Physics, K B D A V College, Nirakarpur, Khurda-752 019 (Orissa), India \\ ${ }^{2}$ Department of Physics, Stewart Science College, Mission Road, Cuttack-753 001 (Orissa), India \\ ${ }^{3}$ Department of Physics, B S College, Daspalla, Nayagarh-752 078 (Orissa), India \\ $\dagger$ Corresponding Author Email: drssd2@yahoo.com
}

(Received November 15, 2009; accepted May 14, 2010)

\begin{abstract}
The objective of this paper is to analyze the effect of mass transfer on unsteady hydromagnetic free convective flow of a viscous incompressible electrically conducting fluid past an infinite vertical porous plate in presence of constant suction and heat source. The governing equations of the flow field are solved using multi parameter perturbation technique and approximate solutions are obtained for velocity field, temperature field, concentration distribution, skin friction and the rate of heat transfer. The effects of the flow parameters such as Hartmann number $M$, Grashof number for heat and mass transfer $G_{r}, G_{c}$; permeability parameter $K_{p}$, Schmidt number $S_{c}$, heat source parameter $S$, Prandtl number $P_{r}$ etc. on the flow field are analyzed with the help of figures and tables. It is observed that a growing Hartmann number or Schmidt number retards the mean velocity as well as the transient velocity of the flow field at all points. The effect of increasing Grashof number for heat and mass transfer or heat source parameter is to accelerate both mean and transient velocity of the flow field at all points. The mean velocity of the flow field increases with an increase in permeability parameter while the transient velocity increases for smaller values of $K_{p}$ $(\leq 1)$ and for higher values the effect reverses. A growing Hartmann number decreases the transient temperature of the flow field at all points while a growing permeability parameter or heat source parameter reverses the effect. The Prandtl number increases the transient temperature for small values of $P_{r}(\leq 1)$ and for higher values the effect reverses. The effect of increasing Schmidt number is to reduce the concentration boundary layer thickness of the flow field at all points. The problem has some relevance in the geophysical and astrophysical studies.
\end{abstract}

Keywords: Hydromagnetic, Mass transfer, Free convection, Porous medium, Suction, Heat source.

\section{INTRODUCTION}

The phenomenon of hydromagnetic flow with heat and mass transfer in an electrically conducting fluid past a porous plate embedded in a porous medium has attracted the attention of a good number of investigators because of its varied applications in many engineering problems such as MHD generators, plasma studies, nuclear reactors, oil exploration, geothermal energy extractions and in the boundary layer control in the field of aerodynamics. Heat transfer in laminar flow is important in problems dealing with chemical reactions and in dissociating fluids.

In view of its wide applications, Hasimoto (1957) initiated the boundary layer growth on a flat plate with suction or injection. Soundalgekar (1974) showed the effect of free convection on steady MHD flow of an electrically conducting fluid past a vertical plate. Yamamoto and Iwamura (1976) explained the flow of a viscous fluid with convective acceleration through a porous medium. Mansutti et al. (1993) have discussed the steady flow of a non-Newtonian fluid past a porous plate with suction or injection. Jha (1998) analyzed the effect of applied magnetic field on transient free convective flow in a vertical channel. Chandran and his associates (1998) have discussed the unsteady free convection flow of an electrically conducting fluid with heat flux and accelerated boundary layer motion in presence of a transverse magnetic field. Acharya et al. (1999) have reported the problem of heat and mass transfer over an accelerating surface with heat source in presence of suction and blowing.

The unsteady free convective MHD flow with heat transfer past a semi-infinite vertical porous moving plate with variable suction has been studied by Kim (2000). Singh and Thakur (2002) have given an exact solution of a plane unsteady MHD flow of a nonNewtonian fluid. Sharma and Pareek (2002) explained the behaviour of steady free convective MHD flow past a vertical porous moving surface. Singh and his co- 
workers (2003) have analyzed the effect of heat and mass transfer in MHD flow of a viscous fluid past a vertical plate under oscillatory suction velocity. Makinde et al. (2003) discussed the unsteady free convective flow with suction on an accelerating porous plate. Sarangi and Jose (2005) studied the unsteady free convective MHD flow and mass transfer past a vertical porous plate with variable temperature. Das and his associates (2006) estimated the mass transfer effects on unsteady flow past an accelerated vertical porous plate with suction employing finite difference analysis. Das et al. (2007) investigated numerically the unsteady free convective MHD flow past an accelerated vertical plate with suction and heat flux. Das and Mitra (2009) discussed the unsteady mixed convective MHD flow and mass transfer past an accelerated infinite vertical plate with suction. Recently, Das and his co-workers (2009) analyzed the effect of mass transfer on MHD flow and heat transfer past a vertical porous plate through a porous medium under oscillatory suction and heat source. More recently, Das et al. (2010) investigated the hydromagnetic convective flow past a vertical porous plate through a porous medium with suction and heat source.

The study of stellar structure on solar surface is connected with mass transfer phenomena. Its origin is attributed to difference in temperature caused by the non-homogeneous production of heat which in many cases can rest not only in the formation of convective currents but also in violent explosions. Mass transfer certainly occurs within the mantle and cores of planets of the size of or larger than the earth. In the present study we therefore, propose to analyze the effect of mass transfer on unsteady free convective flow of a viscous incompressible electrically conducting fluid past an infinite vertical porous plate with constant suction and heat source in presence of a transverse magnetic field. This paper basically highlights the effect of mass transfer on hydromagnetic flow in presence of suction and heat source.

\section{Formulation of the Problem}

Consider the unsteady free convective mass transfer flow of a viscous incompressible electrically conducting fluid past an infinite vertical porous plate in presence of constant suction and heat source and transverse magnetic field. Let the $x^{\prime}$-axis be taken in vertically upward direction along the plate and $y^{\prime}$-axis normal to it. The physical sketch and geometry of the problem is shown in Fig. 1. Neglecting the induced magnetic field and the Joulean heat dissipation and applying Boussinesq's approximation the governing equations of the flow field are given by:

Continuity equation:

$\frac{\partial v^{\prime}}{\partial y^{\prime}}=0 \Rightarrow v^{\prime}=-v_{0}^{\prime}($ Constant $)$,

Momentum equation:

$$
\begin{aligned}
& \frac{\partial u^{\prime}}{\partial t^{\prime}}+v^{\prime} \frac{\partial u^{\prime}}{\partial y^{\prime}}=g \beta\left(T^{\prime}-T_{\infty}^{\prime}\right)+g \beta^{*}\left(C^{\prime}-C_{\infty}^{\prime}\right) \\
& +v \frac{\partial^{2} u^{\prime}}{\partial y^{\prime 2}}-\frac{\sigma B_{0}^{2}}{\rho} u^{\prime}-\frac{v}{K^{\prime}} u^{\prime},
\end{aligned}
$$

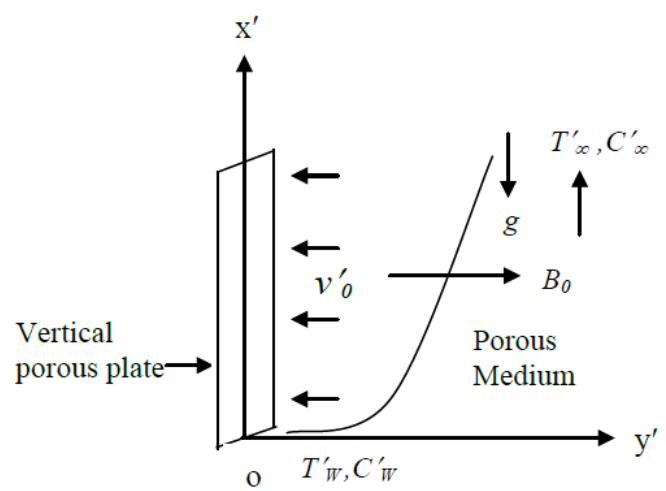

Fig. 1. Physical sketch and geometry of the problem

Energy equation:

$$
\frac{\partial T^{\prime}}{\partial t^{\prime}}+v^{\prime} \frac{\partial T^{\prime}}{\partial y^{\prime}}=k \frac{\partial^{2} T^{\prime}}{\partial y^{\prime 2}}+\frac{v}{C_{p}}\left(\frac{\partial u^{\prime}}{\partial y^{\prime}}\right)^{2}+S^{\prime}\left(T-T_{\infty}^{\prime}\right),
$$

Concentration equation:

$\frac{\partial C^{\prime}}{\partial t^{\prime}}+v^{\prime} \frac{\partial C^{\prime}}{\partial y^{\prime}}=D \frac{\partial^{2} C^{\prime}}{\partial y^{\prime 2}}$.

The boundary conditions of the problem are:

$u^{\prime}=0, v^{\prime}=-v_{0}^{\prime}, T^{\prime}=T_{w}^{\prime}+\varepsilon\left(T_{w}^{\prime}-T_{\infty}^{\prime}\right) e^{i \omega^{\prime} t^{\prime}}$,

$C^{\prime}=C_{w}^{\prime}+\varepsilon\left(C_{w}^{\prime}-C_{\infty}^{\prime}\right) e^{i \omega^{\prime} t^{\prime}}$ at $y^{\prime}=0$,

$u^{\prime} \rightarrow 0, T^{\prime} \rightarrow T_{\infty}^{\prime}, C^{\prime} \rightarrow C_{\infty}^{\prime}$ as $y^{\prime} \rightarrow \infty$.

Introducing the following non-dimensional variables and parameters,

$$
\begin{aligned}
& y=\frac{y v_{0}^{\prime}}{v}, t=\frac{t^{\prime} v_{0}^{\prime 2}}{4 v}, \omega=\frac{4 v \omega^{\prime}}{v_{0}^{\prime 2}}, u=\frac{u^{\prime}}{v_{0}^{\prime}}, v=\frac{\eta_{0}}{\rho}, \\
& M=\left(\frac{\sigma B_{0}^{2}}{\rho}\right) \frac{v}{v_{0}^{\prime 2}}, K_{p}=\frac{v_{0}^{2} K^{\prime}}{v^{2}}, T=\frac{T^{\prime}-T_{\infty}^{\prime}}{T_{w}^{\prime}-T_{\infty}^{\prime}}, \\
& C=\frac{C^{\prime}-C_{\infty}^{\prime}}{C_{w}^{\prime}-C_{\infty}^{\prime}}, P_{r}=\frac{v}{k}, G_{r}=\frac{v g \beta\left(T_{w}^{\prime}-T_{\infty}^{\prime}\right)}{v_{0}^{\prime 3}}, \\
& G_{c}=\frac{v g \beta^{*}\left(C_{w}^{\prime}-C_{\infty}^{\prime}\right)}{v_{0}^{\prime 3}}, S_{c}=\frac{v}{D}, S=\frac{4 S^{\prime} v}{v_{0}^{\prime 2}}, \\
& E_{c}=\frac{v_{0}^{\prime 2}}{C_{p}\left(T_{w}^{\prime}-T_{\infty}^{\prime}\right)},
\end{aligned}
$$

where $g, \rho, \sigma, v, \beta, \beta^{*}, \omega, \eta_{0}, k, T, T_{w}, T_{\infty}, C, C_{w}, C_{\infty}, C_{p}$, $D, P_{r}, S_{c}, G_{r}, G_{c}, S, K_{p}, E_{c}$ and $M$ are respectively the acceleration due to gravity, density, electrical conductivity, coefficient of kinematic viscosity, volumetric coefficient of expansion for heat transfer, volumetric coefficient of expansion for mass transfer, angular frequency, coefficient of viscosity, thermal diffusivity, temperature, temperature at the plate, temperature at infinity, concentration, concentration at the plate, concentration at infinity, specific heat at constant pressure, molecular mass diffusivity, Prandtl number, Schmidt number, Grashof number for heat transfer, Grashof number for mass transfer, heat source parameter, permeability parameter, Eckert number and Hartmann number. 
Substituting Eq. (6) in Eqs. (2), (3) and (4) under boundary conditions (5), we get:

$$
\begin{aligned}
& \frac{1}{4} \frac{\partial u}{\partial t}-\frac{\partial u}{\partial y}=G_{r} T+G_{c} C+\frac{\partial^{2} u}{\partial y^{2}}-\left(M+\frac{1}{K_{p}}\right) u, \\
& \frac{1}{4} \frac{\partial T}{\partial t}-\frac{\partial T}{\partial y}=\frac{1}{P_{r}} \frac{\partial^{2} T}{\partial y^{2}}+\frac{1}{4} S T+E_{c}\left(\frac{\partial u}{\partial y}\right)^{2}, \\
& \frac{1}{4} \frac{\partial C}{\partial t}-\frac{\partial C}{\partial y}=\frac{1}{S_{c}} \frac{\partial^{2} C}{\partial y^{2}} .
\end{aligned}
$$

The corresponding boundary conditions are:

$$
\begin{gathered}
u=0, T=1+\varepsilon e^{i \omega t}, C=1+\varepsilon e^{i \omega t} \text { at } y=0, \\
u \rightarrow 0, T \rightarrow 0, C \rightarrow 0 \text { as } y \rightarrow \infty .
\end{gathered}
$$

\section{METHOD OF SOLUTION}

To solve Eqs. (7), (8) and (9), we assume $\varepsilon$ to be very small and the velocity, temperature and concentration distribution of the flow field in the neighbourhood of the plate as

$$
\begin{aligned}
& u(y, t)=u_{0}(y)+\varepsilon e^{i \omega t} u_{1}(y), \\
& T(y, t)=T_{0}(y)+\varepsilon e^{i \omega t} T_{1}(y), \\
& C(y, t)=C_{0}(y)+\varepsilon e^{i \omega t} C_{1}(y) .
\end{aligned}
$$

Substituting Eqs. (11)-(13) in Eqs. (7)-(9) respectively, equating the harmonic and non-harmonic terms and neglecting the coefficients of $\varepsilon^{2}$, we get Zeroth order:

$u_{0}^{\prime \prime}+u_{0}^{\prime}-\left(M+\frac{1}{K_{p}}\right) u_{0}=-G_{r} T_{0}-G_{c} C_{0}$,

$T_{0}^{\prime \prime}+P_{r} T_{0}^{\prime}+\frac{P_{r} S}{4} T_{0}=-P_{r} E_{c}\left(\frac{\partial u_{0}}{\partial y}\right)^{2}$,

$C_{0}^{\prime \prime}+S_{c} C_{0}^{\prime}=0$

First order:

$$
\begin{aligned}
& u_{1}^{\prime \prime}+u_{1}^{\prime}-\frac{i \omega}{4} u_{1}-\left(M+\frac{1}{K_{p}}\right) u_{1}=-G_{r} T_{1}-G_{c} C_{1}, \\
& T_{1}^{\prime \prime}+P_{r} T_{1}^{\prime}-\frac{P_{r}}{4}(i \omega-S) T_{1}=-2 P_{r} E_{c}\left(\frac{\partial u_{0}}{\partial y}\right)\left(\frac{\partial u_{1}}{\partial y}\right), \\
& C_{1}^{\prime \prime}+S_{c} C_{1}^{\prime}-\frac{i \omega S_{c}}{4} C_{1}=0 .
\end{aligned}
$$

The corresponding boundary conditions are

$$
\begin{aligned}
& y=0: u_{0}=0, T_{0}=1, C_{0}=1, u_{1}=0, T_{1}=1, C_{1}=1 \\
& y \rightarrow \infty: u_{0}=0, T_{0}=0, C_{0}=0, u_{1}=0, T_{1}=0, C_{1}=0
\end{aligned}
$$

Solving Eqs. (16) and (19) under boundary condition (20), we get

$$
\begin{aligned}
& C_{0}=e^{-S_{c} y}, \\
& C_{1}=e^{-m_{1} y},
\end{aligned}
$$

Using multi parameter perturbation technique and assuming $E_{c}<<1$, we assume

$$
\begin{aligned}
& u_{0}=u_{00}+E_{c} u_{01}, \\
& T_{0}=T_{00}+E_{c} T_{01}, \\
& u_{1}=u_{10}+E_{c} u_{11}, \\
& T_{1}=T_{10}+E_{c} T_{11} .
\end{aligned}
$$

Now using Eqs. (23)-(26) in Eqs. (14), (15), (17) and (18) and equating the coefficients of like powers of $E_{\mathrm{c}}$ neglecting those of $E_{\mathrm{c}}{ }^{2}$, we get the following set of differential equations:

Zeroth order:

$$
\begin{aligned}
& u_{00}^{\prime \prime}+u_{00}^{\prime}-\left(M+\frac{1}{K_{p}}\right) u_{00}=-G_{r} T_{00}-G_{c} C_{0}, \\
& u_{10}^{\prime \prime}+u_{10}^{\prime}-\left(M+\frac{1}{K_{p}}+\frac{i \omega}{4}\right) u_{10}=-G_{r} T_{10}-G_{c} C_{1}, \\
& T_{00}^{\prime \prime}+P_{r} T_{00}^{\prime}+\frac{P_{r} S}{4} T_{00}=0, \\
& T_{10}^{\prime \prime}+P_{r} T_{10}^{\prime}-\frac{P_{r}}{4}(i \omega-S) T_{10}=0 .
\end{aligned}
$$

The corresponding boundary conditions are,

$$
\begin{aligned}
& y=0: u_{00}=0, T_{00}=1, u_{10}=0, T_{10}=1 ; \\
& y \rightarrow \infty: u_{00}=0, T_{00}=0, u_{10}=0, T_{10}=0 .
\end{aligned}
$$

First order:

$$
\begin{aligned}
& u_{01}^{\prime \prime}+u_{01}^{\prime}-\left(M+\frac{1}{K_{p}}\right) u_{01}=-G_{r} T_{01}, \\
& u_{11}^{\prime \prime}+u_{11}^{\prime}-\left(M+\frac{1}{K_{p}}+\frac{i \omega}{4}\right) u_{11}=-G_{r} T_{11}, \\
& T_{01}^{\prime \prime}+P_{r} T_{01}^{\prime}+\frac{P_{r} S}{4} T_{01}=-P_{r}\left(u_{00}^{\prime}\right)^{2}, \\
& T_{11}^{\prime \prime}+P_{r} T_{11}^{\prime}-\frac{P_{r}}{4}(i \omega-S) T_{11}=-2 P_{r}\left(\frac{\partial u_{00}}{\partial y}\right)\left(\frac{\partial u_{10}}{\partial y}\right) .
\end{aligned}
$$

The corresponding boundary conditions are,

$$
\begin{aligned}
& y=0: u_{01}=0, T_{01}=0, u_{11}=0, T_{11}=0 ; \\
& y \rightarrow \infty: u_{01}=0, T_{01}=0, u_{11}=0, T_{11}=0 .
\end{aligned}
$$

Solving Eqs. (27)-(30) subject to boundary condition (31), we get

$$
\begin{aligned}
& u_{00}=A_{0} e^{-m_{3} y}+A_{1} e^{-S_{c} y}-A_{2} e^{-m_{7} y}, \\
& T_{00}=e^{-m_{3} y}, \\
& u_{10}=A_{3} e^{-m_{5} y}+A_{4} e^{-m_{1} y}-A_{5} e^{-m_{9} y}, \\
& T_{10}=e^{-m_{5} y} .
\end{aligned}
$$

Solving Eqs. (32)-(35) subject to boundary condition (36), we get

$$
\begin{aligned}
& T_{01}=A_{6} e^{-2 S_{c} y}+A_{7} e^{-2 m_{3} y}+A_{8} e^{-2 m_{5} y}+A_{9} e^{-\left(m_{3}+S_{c}\right) y} \\
& +A_{10} e^{-\left(m_{7}+S_{c}\right) y} \quad+A_{11} e^{-\left(m_{3}+m_{7}\right) y} \quad-A_{12} e^{-m_{3} y}, \\
& T_{11}=A_{13} e^{-\left(m_{3}+m_{5}\right) y}+A_{14} e^{-\left(m_{1}+m_{3}\right) y} \\
& +A_{15} e^{-\left(m_{3}+m_{9}\right) y}+A_{16} e^{-\left(m_{5}+S_{c}\right) y}+A_{17} e^{-\left(m_{1}+S_{c}\right) y} \\
& +A_{18} e^{-\left(m_{9}+S_{c}\right) y}+A_{19} e^{-\left(m_{5}+m_{7}\right) y}+A_{20} e^{-\left(m_{1}+m_{7}\right) y}
\end{aligned}
$$


$+A_{21} e^{-\left(m_{7}+m_{9}\right) y}-A_{22} e^{-m_{5} y}$

(42)

$$
\begin{aligned}
u_{01}= & A_{23} e^{-2 S_{c} y}+A_{24} e^{-2 m_{3} y}+A_{25} e^{-2 m_{7} y} \\
& +A_{26} e^{-\left(m_{3}+S_{c}\right) y}+A_{27} e^{-\left(m_{7}+S_{c}\right) y} \\
& +A_{28} e^{-\left(m_{3}+m_{7}\right) y}+A_{29} e^{-m_{3} y}-A_{30} e^{-m_{7} y}, \\
u_{11}= & A_{31} e^{-\left(m_{3}+m_{5}\right) y}+A_{32} e^{-\left(m_{1}+m_{3}\right) y}+A_{33} e^{-\left(m_{3}+m_{9}\right) y} \\
+ & A_{34} e^{-\left(m_{5}+S_{c}\right) y}+A_{35} e^{-\left(m_{1}+S_{c}\right) y}+A_{36} e^{-\left(m_{9}+S_{c}\right) y} \\
+ & A_{37} e^{-\left(m_{5}+m_{7}\right) y}+A_{38} e^{-\left(m_{1}+m_{7}\right) y}+A_{39} e^{-\left(m_{7}+m_{9}\right) y} \\
+ & A_{40} e^{-m_{5} y}-A_{41} e^{-m_{9} y} .
\end{aligned}
$$

Substituting the values of $C_{0}$ and $C_{1}$ from Eqs. (21) and (22) in Eq. (13) the solution for concentration distribution of the flow field is given by

$C=e^{-S_{c} y}+\varepsilon e^{i \omega t} e^{-m_{1} y}$.

\subsection{Skin Friction}

The skin friction at the wall is given by

$$
\begin{aligned}
\tau_{w}= & \left(\frac{\partial u}{\partial y}\right)_{y=0} \\
= & -m_{3} A_{0}-S_{c} A_{1}+m_{7} A_{2} \\
& -E_{c}\left[2 S_{c} A_{23}+2 m_{3} A_{24}+2 m_{7} A_{25}\right. \\
& +\left(m_{3}+S_{c}\right) A_{26}+\left(m_{7}+S_{c}\right) A_{27} \\
& \left.+\left(m_{3}+m_{7}\right) A_{28}+m_{3} A_{29}-m_{7} A_{30}\right] \\
& -\varepsilon e^{i \omega t} \\
& \left\{m_{5} A_{3}+m_{1} A_{4}-m_{9} A_{5}+E_{c}\left[\left(m_{3}+m_{5}\right) A_{31}\right.\right. \\
& +\left(m_{1}+m_{3}\right) A_{32}+\left(m_{3}+m_{9}\right) A_{33}+\left(m_{5}+S_{c}\right) A_{34} \\
& +\left(m_{1}+S_{c}\right) A_{35}+\left(m_{9}+S_{c}\right) A_{36} \\
& +\left(m_{5}+m_{7}\right) A_{37}+\left(m_{1}+m_{7}\right) A_{38} \\
& \left.\left.+\left(m_{7}+m_{9}\right) A_{39}+m_{5} A_{40}-m_{9} A_{41}\right]\right\}
\end{aligned}
$$

\subsection{Heat Flux}

The heat flux at the wall in terms of Nusselt number is given by

$$
\begin{aligned}
N_{u}= & \left(\frac{\partial T}{\partial y}\right)_{y=0} \\
= & -m_{3}-E_{c}\left[2 S_{c} A_{6}+2 m_{3} A_{7}+2 m_{5} A_{8}\right. \\
& -\left(m_{3}+S_{c}\right) A_{9}+\left(m_{7}+S_{c}\right) A_{10} \\
& \left.+\left(m_{3}+m_{7}\right) A_{11}-m_{3} A_{12}\right] \\
& +\varepsilon e^{i \omega t}\left\{-m_{5}\right. \\
& -E_{c}\left[\left(m_{3}+m_{5}\right) A_{13}+\left(m_{1}+m_{3}\right) A_{14}\right. \\
& +\left(m_{3}+m_{9}\right) A_{15}+\left(m_{5}+S_{c}\right) A_{16}+\left(m_{1}+S_{c}\right) A_{17} \\
& +\left(m_{9}+S_{c}\right) A_{18}+\left(m_{5}+m_{7}\right) A_{19} \\
& \left.\left.+\left(m_{1}+m_{7}\right) A_{20}+\left(m_{7}+m_{9}\right) A_{21}-m_{5} A_{22}\right]\right\}
\end{aligned}
$$

where

$$
\begin{aligned}
& m_{1}=\frac{1}{2}\left[S_{c}+\sqrt{S_{c}^{2}+i \omega S_{c}}\right] \text {, } \\
& m_{2}=\frac{1}{2}\left[-S_{c}+\sqrt{S_{c}^{2}+i \omega S_{c}}\right] \text {, } \\
& m_{3}=\frac{1}{2}\left[P_{r}+\sqrt{P_{r}^{2}-S P_{r}}\right] \text {, } \\
& m_{4}=\frac{1}{2}\left[-P_{r}+\sqrt{P_{r}^{2}-S P_{r}}\right] \text {, } \\
& m_{5}=\frac{1}{2}\left[P_{r}+\sqrt{P_{r}^{2}-P_{r}(S-i \omega)}\right] \text {, } \\
& m_{6}=\frac{1}{2}\left[-P_{r}+\sqrt{P_{r}^{2}-P_{r}(S-i \omega)}\right] \text {, } \\
& m_{7}=\frac{1}{2}\left[1+\sqrt{1+4\left(M+\frac{1}{K_{p}}\right)}\right] \text {, } \\
& m_{8}=\frac{1}{2}\left[-1+\sqrt{1+4\left(M+\frac{1}{K_{p}}\right)}\right] \text {, } \\
& m_{9}=\frac{1}{2}\left[1+\sqrt{1+4\left(M+\frac{1}{K_{p}}+\frac{i \omega}{4}\right)},\right. \\
& m_{10}=\frac{1}{2}\left[-1+\sqrt{1+4\left(M+\frac{1}{K_{p}}+\frac{i \omega}{4}\right)}\right], \\
& A_{0}=\frac{G_{r}}{\left(m_{7}-m_{3}\right)\left(m_{8}+m_{3}\right)} \text {, } \\
& A_{1}=\frac{G_{c}}{\left(m_{7}-S_{c}\right)\left(m_{8}+S_{c}\right)}, A_{2}=A_{0}+A_{1}, \\
& A_{3}=\frac{G_{r}}{\left(m_{9}-m_{5}\right)\left(m_{10}+m_{5}\right)} \\
& A_{4}=\frac{G_{c}}{\left(m_{9}-m_{1}\right)\left(m_{10}+m_{1}\right)}, \\
& A_{5}=A_{3}+A_{4}, A_{6}=\frac{P_{r} S_{c}^{2} A_{1}^{2}}{\left(m_{3}-2 S_{c}\right)\left(m_{4}+2 S_{c}\right)}, \\
& A_{7}=\frac{-P_{r} m_{3} A_{0}^{2}}{\left(m_{4}+2 m_{3}\right)}, A_{8}=\frac{P_{r} m_{7}^{2} A_{2}^{2}}{\left(m_{3}-2 m_{7}\right)\left(m_{4}+2 m_{7}\right)}, \\
& A_{9}=-\frac{2 A_{0} A_{1} m_{3} P_{r}}{\left(m_{3}+m_{4}+S_{c}\right)}, \\
& A_{10}=-\frac{2 P_{r} S_{c} A_{1} A_{2} m_{7}}{\left(m_{3}-m_{7}-S_{c}\right)\left(m_{4}+m_{7}+S_{c}\right)}, \\
& A_{11}=\frac{2 P_{r} A_{0} A_{1} m_{3}}{\left(m_{3}+m_{4}+m_{7}\right)}, \\
& A_{12}=A_{6}+A_{7}+A_{8}+A_{9}+A_{10}+A_{11} \text {, } \\
& A_{13}=-\frac{2 P_{r} A_{0} A_{5}}{\left(m_{3}+m_{5}+m_{6}\right)}, \\
& A_{14}=\frac{2 P_{r} A_{0} A_{4} m_{1} m_{3}}{\left(m_{5}-m_{3}-m_{1}\right)\left(m_{6}+m_{3}+m_{1}\right)} \text {, } \\
& A_{15}=\frac{2 P_{r} A_{0} A_{5} m_{3} m_{9}}{\left(m_{9}-m_{5}+m_{3}\right)\left(m_{9}+m_{6}+m_{3}\right)}, \\
& A_{16}=-\frac{2 P_{r} A_{1} A_{3} m_{5}}{\left(m_{6}+m_{5}+S_{c}\right)} \text {, }
\end{aligned}
$$




$$
\begin{aligned}
& A_{17}=\frac{2 P_{r} S_{c} A_{1} A_{4} m_{1}}{\left(m_{5}-m_{1}-S_{c}\right)\left(m_{6}+m_{1}+S_{c}\right)}, \\
& A_{18}=\frac{2 P_{r} S_{c} A_{1} A_{5} m_{9}}{\left(m_{9}-m_{5}+S_{c}\right)\left(m_{9}+m_{6}+S_{c}\right)}, \\
& A_{19}=\frac{2 P_{r} A_{2} A_{3} m_{5}}{\left(m_{7}+m_{6}+m_{5}\right)}, \\
& A_{20}=\frac{2 P_{r} A_{2} A_{4} m_{1} m_{7}}{\left(m_{7}-m_{5}+m_{1}\right)\left(m_{7}+m_{6}+m_{1}\right)}, \\
& A_{21}=\frac{2 P_{r} A_{2} A_{5} m_{7} m_{9}}{\left(m_{9}+m_{7}+m_{5}\right)\left(m_{9}+m_{7}+m_{6}\right)}, \\
& A_{22}=A_{13}+A_{14}+A_{15}+A_{16}+A_{17} \\
& +A_{18}+A_{19}+A_{20}+A_{21} \text {, } \\
& A_{23}=\frac{G_{r} A_{6}}{\left(m_{7}-2 S_{c}\right)\left(m_{8}+2 S_{c}\right)}, \\
& A_{24}=\frac{G_{r} A_{7}}{\left(m_{7}-2 m_{3}\right)\left(m_{8}+2 m_{3}\right)}, \\
& A_{25}=\frac{-G_{r} A_{8}}{m_{7}\left(m_{8}+2 m_{7}\right)}, \\
& A_{26}=\frac{G_{r} A_{9}}{\left(m_{7}-m_{3}-S_{c}\right)\left(m_{8}+m_{3}+S_{c}\right)}, \\
& A_{27}=\frac{-G_{r} A_{10}}{S_{c}\left(m_{8}+m_{7}+S_{c}\right)}, \\
& A_{28}=\frac{-G_{r} A_{11}}{m_{3}\left(m_{8}+m_{7}+m_{3}\right)}, \\
& A_{29}=\frac{G_{r} A_{12}}{\left(m_{3}-m_{7}\right)\left(m_{8}+m_{3}\right)}, \\
& A_{30}=A_{23}+A_{24}+A_{25}+A_{26}+A_{27}+A_{28}+A_{29} \text {, } \\
& A_{31}=\frac{G_{r} A_{13}}{\left(m_{9}-m_{5}-m_{3}\right)\left(m_{10}+m_{5}+m_{3}\right)} \text {, } \\
& A_{32}=\frac{G_{r} A_{14}}{\left(m_{9}-m_{3}-m_{1}\right)\left(m_{10}+m_{3}+m_{1}\right)} \text {, } \\
& A_{33}=\frac{-G_{r} A_{15}}{m_{3}\left(m_{10}+m_{9}+m_{3}\right)}, \\
& A_{34}=\frac{G_{r} A_{16}}{\left(m_{9}-m_{5}-S_{c}\right)\left(m_{10}+m_{5}+S_{c}\right)}, \\
& A_{35}=\frac{G_{r} A_{17}}{\left(m_{9}-m_{1}-S_{c}\right)\left(m_{10}+m_{1}+S_{c}\right)} \text {, } \\
& A_{36}=\frac{-G_{r} A_{18}}{S_{c}\left(m_{10}+m_{9}+S_{c}\right)}, \\
& A_{37}=\frac{G_{r} A_{19}}{\left(m_{9}-m_{7}-m_{5}\right)\left(m_{10}+m_{7}+m_{5}\right)}, \\
& A_{38}=\frac{G_{r} A_{20}}{\left(m_{9}-m_{7}-m_{1}\right)\left(m_{10}+m_{7}+m_{1}\right)} \text {, } \\
& A_{39}=\frac{-G_{r} A_{21}}{m_{7}\left(m_{10}+m_{9}+m_{7}\right)}, \\
& A_{40}=\frac{G_{r} A_{22}}{\left(m_{9}-m_{5}\right)\left(m_{10}+m_{5}\right)}, \\
& A_{41}=A_{31}+A_{32}+A_{33}+A_{34}+A_{35} \\
& +A_{36}+A_{37}+A_{38}+A_{39}+A_{40} \text {. }
\end{aligned}
$$

\section{ReSUlts AND Discussions}

The effect of mass transfer on unsteady free convective flow of a viscous incompressible electrically conducting fluid past an infinite vertical porous plate with constant suction and heat source in presence of a transverse magnetic field has been studied. The governing equations of the flow field are solved employing multi parameter perturbation technique and approximate solutions are obtained for velocity field, temperature field, concentration distribution, skin friction and rate of heat transfer. The effects of the pertinent parameters on the flow field are analyzed and discussed with the help of velocity profiles (Figs. 2-13), temperature profiles (Figs. 14-17), concentration distribution (Fig. 18) and Tables 1-5. To be more realistic, during numerical calculations we have chosen the values of $\mathrm{P}_{\mathrm{r}}=0.71$ representing air at $20^{\circ} \mathrm{C}, \mathrm{S}_{\mathrm{c}}=0.60$ representing $\mathrm{H}_{2} \mathrm{O}$ vapour, $\mathrm{G}_{\mathrm{r}}>0$ corresponding to cooling of the plate and $\mathrm{S}>0$ representing heat source.

\subsection{Velocity Field}

The velocity of the flow field is found to change more or less with the variation of the flow parameters. The major factors affecting the velocity of the flow field are Hartmann number $M$, permeability parameter $K_{p}$, Grashof number for heat and mass transfer $G_{r}, G_{c}$; Schmidt number $S_{c}$, heat source parameter $S$ and Prandtl number $P$. The effects of these parameters on the velocity field have been analyzed with the help of Figs. 2-13. The velocity profiles closely agree with those of Das et al. (2010).

Figures 2 and 8 depict the effect of magnetic field on mean and transient velocity of the flow field respectively. It is observed that a growing Hartmann number decelerates both mean and transient velocity of the flow field at all points due to the magnetic pull of the Lorentz force acting on the flow field. The effects of permeability parameter on mean and transient velocity of the flow field are shown in Figs. 3 and 9 respectively.

The permeability parameter is found to enhance the mean velocity of the flow field at all points while the transient velocity increases for small values of $K_{p}\left(K_{p} \leq 1\right)$ and the effect reverses for higher values. Figures 4 and 10 show the effect of Grashof number for heat transfer on mean and transient velocity respectively. The Grashof number for heat transfer is found to enhance both mean and transient velocity at all points due to the action of free convection current in the flow field. Figures 5 and 11 present the effect of Grashof number for mass transfer on mean and transient velocity respectively. Both the figures show the accelerating effect of the parameter on the velocity of the flow field at all points. Figures 6 and 12 discuss the effect of heat source parameter on the velocity of the flow field. In both mean and transient velocity of the flow field a growing heat source parameter enhances the effect at all points. In Figs. 8 and 13, we depict the effect of mass transfer on mean and transient velocity of the flow field respectively. The presence of heavier diffusing species in the flow field is found to decelerate both mean and transient velocity at all points. 


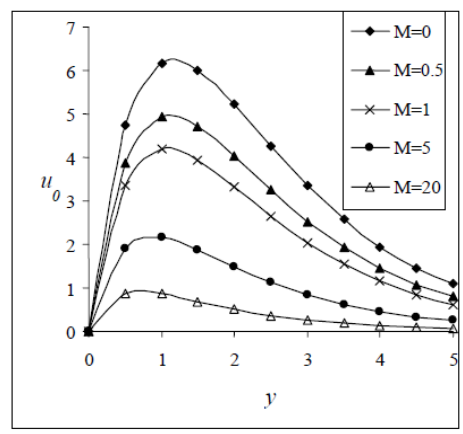

Fig. 2. Mean velocity profiles against $y$ for different values of $M$ with $G_{r}=5, G_{c}=5, E_{c}=0.002, \omega=5.0, \varepsilon=0.2, \omega t=\pi / 2$, $K_{p}=1, S=0.1, S_{c}=0.60, P_{r}=0.71$

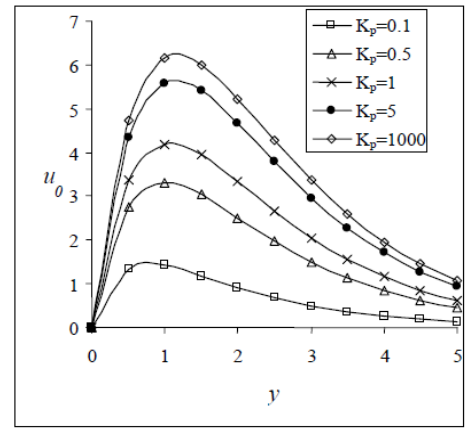

Fig. 3. Mean velocity profiles against $y$ for different values of $K_{p}$ with $G_{r}=5, G_{c}=5, M=1, E_{c}=0.002, \omega=5.0, \varepsilon=0.2$, $\omega t=\pi / 2, \mathrm{~S}=0.1, S_{c}=0.60, P_{r}=0.71$

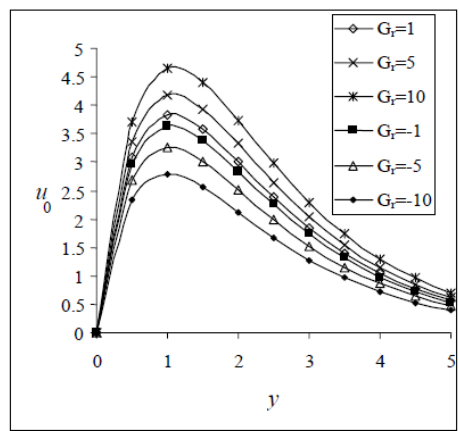

Fig. 4. Mean velocity profiles against $y$ for different values of $G_{r}$ with $G_{c}=5, M=1, E_{c}=0.002, \omega=5.0, \varepsilon=0.2, \omega t=\pi / 2$, $K_{p}=1, S=0.1, S_{c}=0.60, P_{r}=0.71$

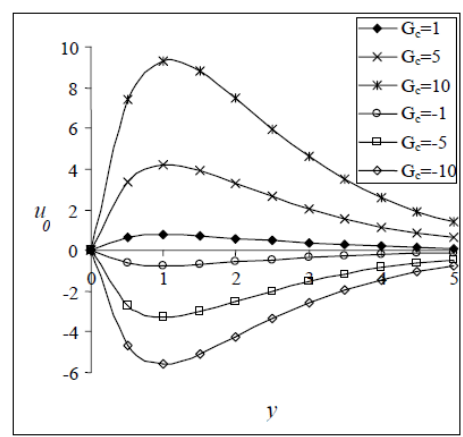

Fig. 5. Mean velocity profiles against $y$ for different values of $G_{c}$ with $G_{r}=5, M=1, E_{c}=0.002, \omega=5.0, \varepsilon=0.2, \omega t=\pi / 2$, $K_{p}=1, S=0.1, S_{c}=0.60, P_{r}=0.71$

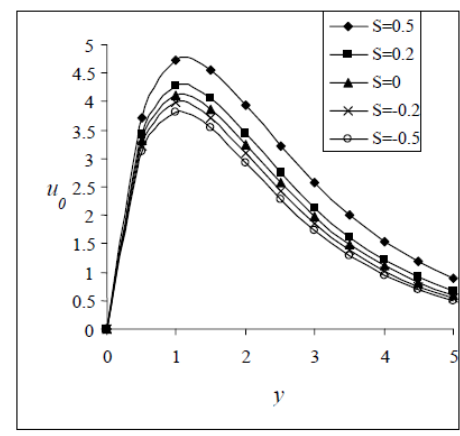

Fig. 6. Mean velocity profiles against $y$ for different values of $S$ with $G_{r}=5, G_{c}=5, M=1, E_{c}=0.002, \omega=5.0$, $\varepsilon=0.2, \omega t=\pi / 2, K_{p}=1, \mathrm{Sc}=0.60, P_{r}=0.71$

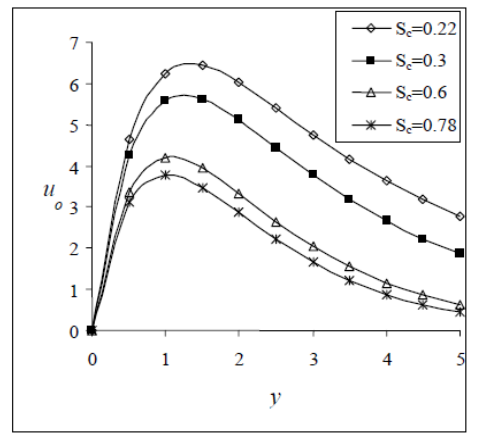

Fig. 7. Mean velocity profiles against $y$ for different values of $S_{c}$ with $G_{r}=5, G_{c}=5, M=1, E_{c}=0.002, \omega=5.0$, $\varepsilon=0.2, \omega t=\pi / 2, K_{p}=1, S=0.1, P_{r}=0.71$

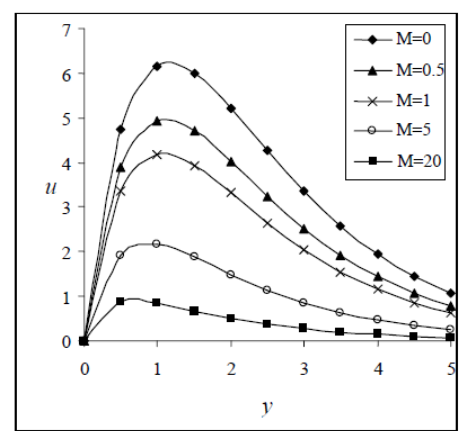

Fig. 8. Transient velocity profiles against $y$ for different values of $M$ with $G_{r}=5, G_{c}=5, E_{c}=0.002, \omega=5.0, \varepsilon=0.2$, $\omega t=\pi / 2, K_{p}=1, S=0.1, S_{c}=0.60, P_{r}=0.71$

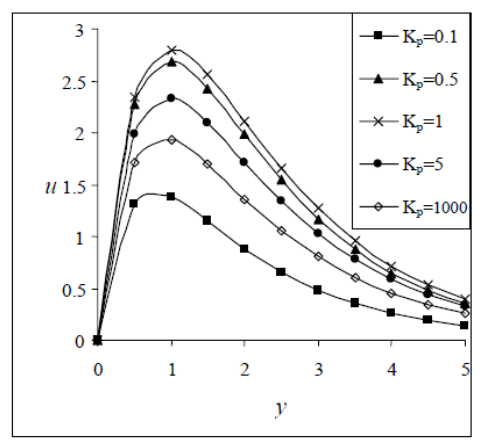

Fig. 9. Transient velocity profiles against $y$ for different values of $K_{p}$ with $G_{r}=5, G_{c}=5, E_{c}=0.002, S=0.1$, $S_{c}=0.60, P_{r}=0.71, M=1, \omega=5.0, \varepsilon=0.2, \omega t=\pi / 2$ 


\subsection{Temperature Field}

The temperature of the flow field suffers a substantial change with the variation of the flow parameters such as Prandtl number $P_{r}$, Hartmann number $M$, heat source parameter $S$ and permeability parameter $K_{p}$. These variations are shown in Figs. 14-17. The temperature profiles are in good agreement with those of Das et al. (2010).

Figure 14 depicts the effect of Prandtl number on the temperature field keeping other parameters of the flow field constant. It is interesting to observe that for lower value of $\operatorname{Pr}(\leq 1)$, it enhances the transient temperature while for higher values the effect reverses. Figure 15 shows the effect of magnetic parameter on the temperature field. The effect of Hartmann number is to retard the temperature of the flow field at all points. Curve with $M=0$ corresponds to the non-MHD flow. This shows that in absence of magnetic field the temperature first rises near the plate and thereafter, it falls. In other curves there is a decrease in temperature at all points. This shows the dominating effect of the magnetic field due to the action of the Lorentz force in the flow field. The effect of heat source parameter on the temperature field is presented in Fig. 16. The heat source parameter is found to enhance the temperature of the flow field at all points. In Fig. 17, we analyze the effect of permeability parameter on the temperature field. A growing permeability parameter is found to increase the temperature of the flow field at all points.

\subsection{Concentration Distribution}

The variation in the concentration boundary layer of the flow field is shown in Fig. 18 due to the change in the Schmidt number $S_{c}$. Curves with $S_{c}=0.22,0.30,0.6$ and 0.78 respectively, represent the concentration distribution in presence of $\mathrm{H}_{2}, \mathrm{He}, \mathrm{H}_{2} \mathrm{O}$ vapour and $\mathrm{NH}_{3}$ in the flow field. Comparing the curves of the said figure it is observed that a growing Schmidt number decreases the concentration boundary layer thickness of the flow field at all points.

\subsection{Skin Friction}

The values of skin friction at the wall against $K_{p}$ for different values of Hartmann number $M$ and heat source parameter $S$ are entered in Tables 1 and 2 respectively. From Table 1, it is observed that a growing Hartmann number $M$ reduces the skin friction at the wall for a fixed value of the permeability parameter due to the action of Lorentz force in the flow field. It is further observed from Table 2 that both permeability parameter $K_{p}$ and heat source parameter $S$ enhance the skin friction at the wall. Our observation for skin friction agrees with those of Das et al. (2010).

\subsection{Rate of Heat Transfer}

The rate of heat transfer at the wall varies with the variation of Prandtl number $P_{r}$, Hartmann number $M$, permeability parameter $K_{p}$. These variations are entered in the Tables 3-5. From Table 3, we observe that a growing Prandtl number or permeability parameter increase the magnitude of the rate of heat transfer at the wall. Further, it is observed from Table 4 that an increase in Hartmann number reduces its value for a given value of Prandtl number. Again from Table 5, we see that for a given value of permeability parameter it enhances the magnitude of rate of heat transfer for small values of $M$ and for higher values the effect reverses due to the magnetic pull of the Lorentz force acting on the flow field. These variations agree with those of Das et al. (2010) with a little deviation for higher value of $M$.

\section{CONClusions}

We summarize below the following results of physical interest on the velocity, temperature and the concentration distribution of the flow field and also on the wall shear stress and rate of heat transfer at the wall.

1. A growing Hartmann number or Schmidt number retards the mean velocity as well as the transient velocity of the flow field at all points.

2. The effect of increasing Grashof number for heat and mass transfer or heat source parameter is to accelerate both mean and transient velocity of the flow field at all points.

3. The mean velocity of the flow field increases with an increase in permeability parameter while the transient velocity increases for smaller values of $K_{p}(\leq 1)$ and for higher values the effect reverses.

4. A growing Hartmann number decreases transient temperature of the flow field at all points while a growing permeability parameter or heat source parameter reverses the effect.

5. The Prandtl number $P_{r}$ increases the transient temperature of the flow field at all points for small values of $P_{r}(\leq 1)$ and for higher values the effect reverses.

6. The effect of increasing Schmidt number is to reduce the concentration boundary layer thickness of the flow field at all points.

7. A growing Hartmann number reduces the skin friction at the wall while a growing permeability parameter or heat source parameter reverses the effect.

8. The effect of increasing Prandtl number or permeability parameter is to increase the magnitude of the rate of heat transfer at the wall. On the other hand, a growing Hartmann number reduces its value for a given value of Prandtl number while for a given value of permeability parameter it enhances the magnitude of rate of heat transfer for small values of $M$ and for higher values the effect reverses due to the magnetic pull of the Lorentz force acting on the flow field. 


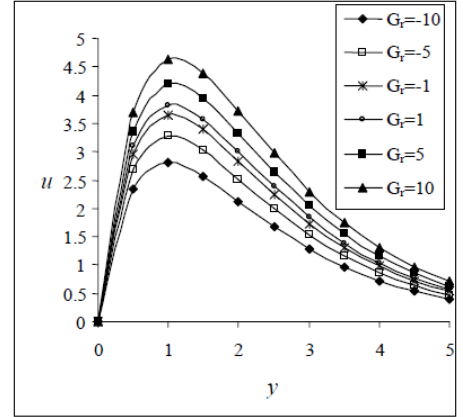

Fig. 10. Transient velocity profiles against $y$ for different values of $G_{r}$ with $G_{c}=5, M=1, E_{c}=0.002, \omega=5.0, \varepsilon=0.2$, $\omega t=\pi / 2, K_{p}=1, S=0.1, S_{c}=0.60, P_{r}=0.71$

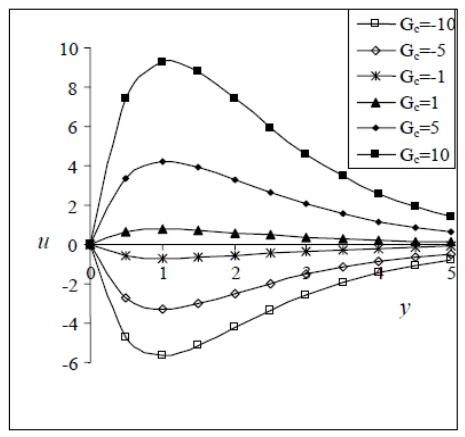

Fig. 11. Transient velocity profiles against $y$ for different values of $G_{c}$ with $G_{r}=5, M=1, E_{c}=0.002, \omega=5.0, \varepsilon=0.2$, $\omega t=\pi / 2, K_{p}=1, S=0.1, S_{c}=0.60, P_{r}=0.71$

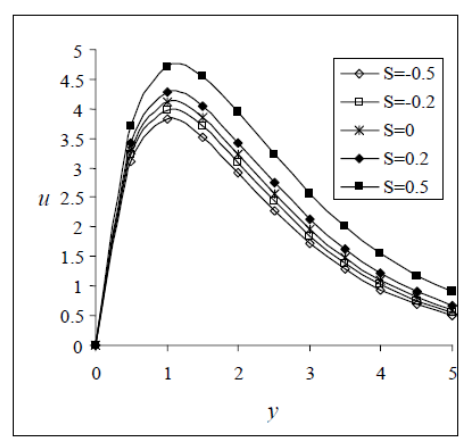

Fig. 12. Transient velocity profiles against $y$ for different values of $S$ with $G_{r}=5, G_{c}=5, E_{c}=0.002, M=1, K_{p}=1$, $S_{c}=0.60, P_{r}=0.71, \omega=5.0, \varepsilon=0.2, \omega t=\pi / 2$

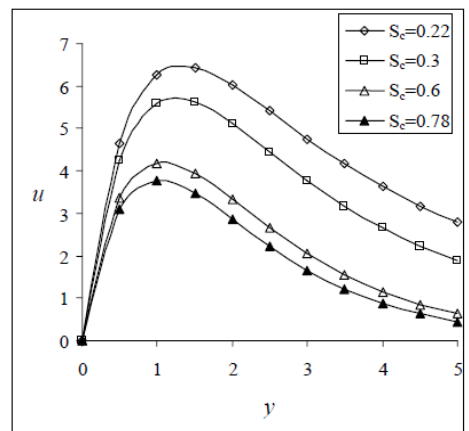

Fig. 13. Transient velocity profiles against $y$ for different values of $S_{c}$ with $G_{r}=5, G_{c}=5, E_{c}=0.002, M=1, K_{p}=1$, $S=0.1, P_{r}=0.71, \omega=5.0, \varepsilon=0.2, \omega t=\pi / 2$

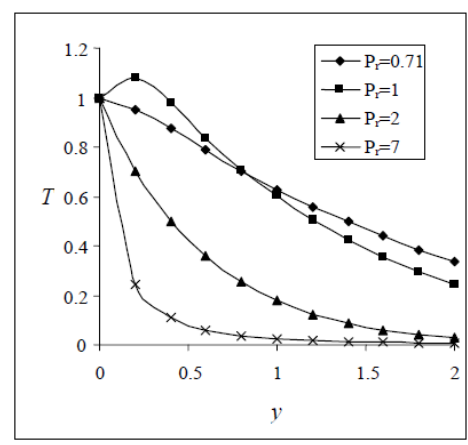

Fig. 14. Temperature profiles against $y$ for different values of $P_{r}$ with $G_{r}=5, G_{c}=5, M=1, E_{c}=0.002, \omega=5.0$, $\varepsilon=0.2, \omega t=\pi / 2, K_{p}=1, S=0.1$

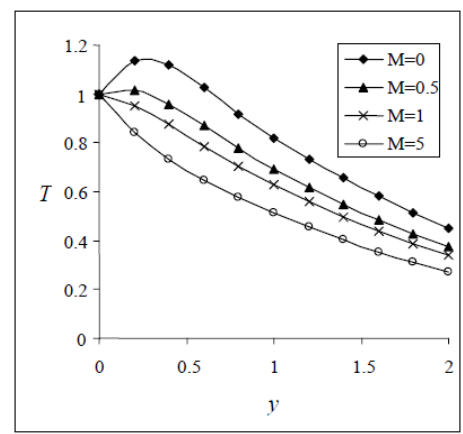

Fig. 15. Temperature profiles against $y$ for different values of $M$ with $G_{r}=5, G_{c}=5, E_{c}=0.002, \omega=5.0, \varepsilon=0.2$, $\omega t=\pi / 2, K_{p}=1, S=0.1, P_{r}=0.71$

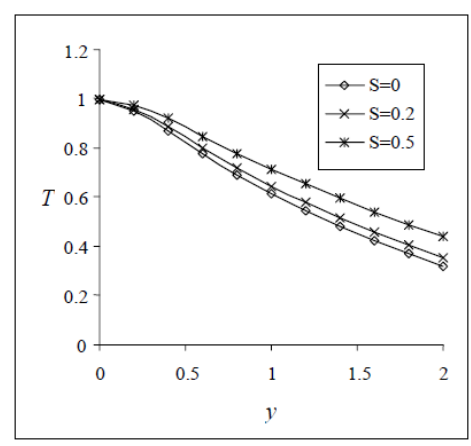

Fig. 16. Temperature profiles against $y$ for different values of $S$ with $G_{r}=5, G_{c}=5, M=1, E_{c}=0.002, \omega=5.0$, $\varepsilon=0.2, \omega t=\pi / 2, K_{p}=1, P_{r}=0.71$

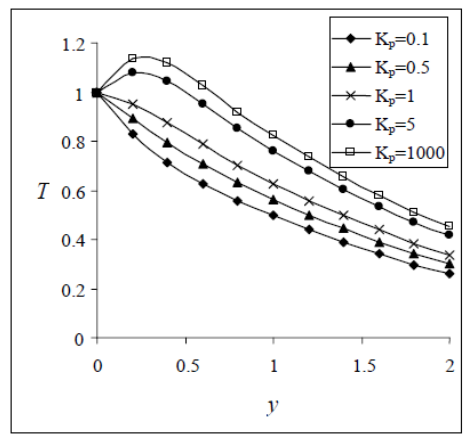

Fig. 17. Temperature profiles against $y$ for different values of $K_{p}$ with $G_{r}=5, G_{c}=5, M=1, E_{c}=0.002, \omega=5.0$, $\varepsilon=0.2, \omega t=\pi / 2, S=0.1, P_{r}=0.71$ 


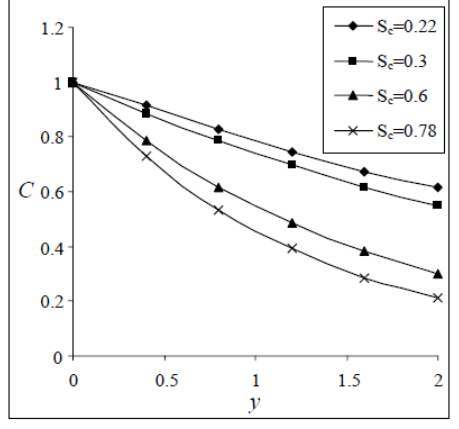

Fig. 18. Concentration profiles against $y$ for different values of $S_{c}$ with $\omega=5.0, \varepsilon=0.2, \omega t=\pi / 2$

\section{REFERENCES}

Acharya, M., L.P. Singh and G.C. Dash (1999). Heat and mass transfer over an accelerating surface with heat source in presence of suction and blowing. Int. J.Engng. Sci. 37, 1-18.

Chandran, P., N.C. Sacheti and A.K. Singh (1998). Unsteady hydromagnetic free convection flow with heat flux and accelerated boundary motion. $J$. Phys. Soc. Japan 67, 124-129.

Das, S.S., S.K. Sahoo and G.C. Dash (2006). Numerical solution of mass transfer effects on unsteady flow past an accelerated vertical porous plate with suction. Bull. Malays. Math. Sci. Soc. 29(1), 3342.

Das, S.S., A. Satapathy, J.K. Das and S.K. Sahoo (2007). Numerical solution of unsteady free convective MHD flow past an accelerated vertical plate with suction and heat flux. J. Ultra Sci. Phys. Sci. 19(1), 105-112.

Das, S.S. and M. Mitra (2009).Unsteady mixed convective MHD flow and mass transfer past an accelerated infinite vertical plate with suction. Ind. J. Sci. Tech. 2 (5), 18-22.

Das, S.S., A. Satapathy, J.K. Das and J.P. Panda (2009). Mass transfer effects on MHD flow and heat transfer past a vertical porous plate through a porous medium under oscillatory suction and heat source. Int. J. Heat Mass Transfer 52, 5962-5969.

Das S.S., U.K. Tripathy and J.K. Das (2010). Hydromagnetic convective flow past a vertical porous plate through a porous medium with suction and heat source. International Journal of Energy and Environment 1(3), 467-478.

Hasimoto, H. (1957). Boundary layer growth on a flat plate with suction or injection. J. Phys. Soc. Japan $12,68-72$.

Jha, B.K. (1998). Effects of applied magnetic field on transient free convective flow in a vertical channel. Ind. J. Pure Appl. Math. 29(4), 441-445.
Kim, Y.J. (2000). Unsteady MHD convective heat transfer past a semi-infinite vertical porous moving plate with variable suction. Int. J. Engng. Sci. 38, 833-845.

Makinde, O.D., J.M. Mango and D.M. Theuri (2003). Unsteady free convection flow with suction on an accelerating porous plate. AMSE J. Mod. Meas. Cont., B 72 (3), 39-46.

Mansutti, D., G. Pontrelli and K.R. Rajagopal (1993). Steady flows of non-Newtonian fluids past a porous plate with suction or injection. Int. J. Num. Methods Fluids 17, 927-941.

Sarangi, K.C. and C.B. Jose (2005). Unsteady free convective MHD flow and mass transfer past a vertical porous plate with variable temperature. Bull. Cal. Math. Soc. 97 (2), 137-146.

Sharma, P.R. and D. Pareek (2002). Steady free convection MHD flow past a vertical porous moving surface. Ind. J. Theo. Phys. 50, 5-13.

Singh, A.K., A.K. Singh and N.P. Singh (2003). Heat and mass transfer in MHD flow of a viscous fluid past a vertical plate under oscillatory suction velocity. Ind. J. Pure Appl. Math. 34(3), 429-442.

Singh, B. and C. Thakur (2002). An exact solution of plane unsteady MHD non-Newtonian fluid flows. Ind. J. Pure Appl. Math. 33(7), 993 -1001.

Soundalgekar, V.M. (1974). Free convection effects on steady MHD flow past a vertical porous plate. $J$. Fluid Mech. 66, 541-551.

Yamamoto, K. and N. Iwamura (1976). Flow with convective acceleration through a porous medium. Engng. Math. 10, 41-54. 
S.S. Das et al. / JAFM, Vol. 4, No. 4, pp. 91-100, 2011.

Table 1 Values of skin friction ( $\tau$ ) at the wall against $K_{p}$ for different values of $M$ with $G_{r}=5, G_{c}=5$,

$E_{c}=0.002, \omega=5.0, \varepsilon=0.2, \omega t=\pi / 2, S=0.1, S_{c}=0.60$
Table 3 Values of rate of heat transfer $\left(N_{u}\right)$ at the wall against $P_{r}$ for different values of $K_{p}$ with $G_{r}=5, G_{c}=5, M=1$, $E_{c}=0.002, \omega=5.0, \varepsilon=0.2, \omega t=\pi / 2, S=0.1, S_{c}=0.60$

\begin{tabular}{|c|c|c|c|c|c|c|c|c|c|}
\hline \multirow{2}{*}{$K_{p}$} & \multicolumn{4}{|c|}{$\tau$} & \multirow{2}{*}{$P_{r}$} & \multicolumn{4}{|c|}{$N_{u}$} \\
\hline & $M=0$ & $M=0.1$ & $M=5$ & $M=20$ & & $K_{p}=0.1$ & $K_{p}=0.5$ & $K_{p}=10$ & $K_{p}=1000$ \\
\hline 0.1 & 5.653382 & 5.534346 & 4.728732 & 3.473894 & 0.71 & -0.22261 & 0.25378 & 1.62899 & 1.84067 \\
\hline 0.5 & 10.02986 & 9.805144 & 6.488265 & 3.995386 & 1 & -0.37971 & 0.62512 & 5.88600 & 6.94199 \\
\hline 5 & 14.57578 & 14.11291 & 7.273244 & 4.150112 & 7 & -5.24947 & -6.50457 & -9.07674 & -9.39557 \\
\hline 10 & 15.09217 & 14.57578 & 7.326112 & 4.159260 & 9 & -6.64083 & -7.97398 & -10.7089 & -11.04849 \\
\hline 1000 & 15.66754 & 15.08670 & 7.379662 & 4.168377 & 11 & -8.06782 & -9.50515 & -12.4663 & -12.83434 \\
\hline
\end{tabular}

Table 2 Values of skin friction $(\tau)$ at the wall against $K_{p}$ for different values of $S$ with $G_{r}=5, G_{c}=5, E_{c}=0.002, \omega=5.0, \varepsilon=0.2, \omega t=\pi / 2, S_{c}=0.60$

\begin{tabular}{ccccccc}
\hline \multirow{2}{*}{$K_{p}$} & \multicolumn{7}{c}{$\tau$} \\
\cline { 2 - 6 } & $S=-0.5$ & $S=-0.2$ & $S=-0.1$ & $S=0.1$ & $S=0.2$ & $S=0.5$ \\
\hline 0.1 & 5.12145 & 5.24706 & 5.29364 & 5.39647 & 5.45411 & 5.67230 \\
\hline 0.5 & 8.30214 & 8.53049 & 8.61904 & 8.82282 & 8.94266 & 9.44074 \\
\hline 1 & 9.40552 & 9.66218 & 9.7637 & 10.00108 & 10.14313 & 10.75373 \\
\hline 5 & 10.62662 & 10.95844 & 11.09 & 11.39881 & 11.58467 & 12.39587 \\
\hline 10 & 10.82795 & 11.1691 & 11.30463 & 11.62338 & 11.81564 & 12.65832 \\
\hline 1000 & 11.04011 & 11.3912 & 11.53098 & 11.86037 & 12.05950 & 12.93613 \\
\hline
\end{tabular}

Table 4 Values of rate of heat transfer $\left(N_{u}\right)$ at the wall against $P_{r}$ for different values of $M$ with $G_{r}=5, G_{c}=5, M=1$, $E_{c}=0.002, \omega=5.0, \varepsilon=0.2, \omega t=\pi / 2, S=0.1, S_{c}=0.60$
Table 5 Values of rate of heat transfer $\left(N_{u}\right)$ at the wall against $K_{p}$ for different values of $M$ with $G_{r}=5, G_{c}=5$, $M=1, E_{c}=0.002, \omega=5.0, \varepsilon=0.2, \omega t=\pi / 2, S=0.1, S_{c}=0.60$

\begin{tabular}{|c|c|c|c|c|c|c|c|c|c|}
\hline \multirow{2}{*}{$P_{r}$} & \multicolumn{4}{|c|}{$N_{u}$} & \multirow{2}{*}{$K_{p}$} & \multicolumn{4}{|c|}{$N_{u}$} \\
\hline & $M=0$ & $M=0.1$ & $M=5$ & $M=20$ & & $M=0$ & $M=0.1$ & $M=5$ & $M=20$ \\
\hline 0.71 & 1.84299 & 1.62899 & -0.32462 & -0.25617 & 0.1 & -0.21328 & -0.21433 & -0.24373 & -0.26289 \\
\hline 1 & 6.95398 & 5.88600 & -0.47670 & -0.44308 & 0.5 & 0.60664 & 0.54699 & -0.16004 & -0.25733 \\
\hline 7 & -9.39900 & -9.07674 & -5.62259 & -5.10180 & 5 & 6.32474 & 5.23180 & -0.08245 & -0.25511 \\
\hline 9 & -11.0522 & -10.70889 & -7.03978 & -6.44508 & 10 & 7.77240 & 6.32474 & -0.07593 & -0.25497 \\
\hline 11 & -12.8383 & -12.46632 & -8.49695 & -7.85540 & 1000 & 9.72173 & 7.75568 & -0.06914 & -0.25483 \\
\hline
\end{tabular}

